\title{
LA SEMANTICA DEI NOMI PROPRI AUGURALI
}

\author{
Denisa-Alexandra Ionescu \\ Università „Babeș-Bolyai”, Cluj-Napoca, Romania
}

\begin{abstract}
Semantics of augural proper names
Abstract: The present approach aims at delineating the general framework of the semantics of proper names, thus allowing one to focus subsequently on the particular case of augural names. Our research explores the meaning of anthroponyms, so that it can consequently compare, from the viewpoint of their content, the different types of proper names in relation to the particular case of augural names.

Given their motivation, which is most of the times sacred, Christian, inspired by the Divinity, augural proper names represent the most salient embodiment of the guiding red thread that semantics represents with respect to the interpretation of proper names in general.
\end{abstract}

Keywords: anthroponym, semantics, meaning, reference, motivation.

A vedere tale titolo una domanda potrebbe sorgere: perché lo studio di una categoria così specifica e condizionante come quella dei nomi augurali? Perché non una classe più ampia di antroponimi che fornisca un materiale di investigazione molto più ricco? A mio parere, tranne i soprannomi, questi antroponimi dal carattere assai particolare conservano ancora visibile la loro motivazione originaria e, di conseguenza, il loro senso. In più, le speranze, i cosiddetti auguri che si fanno al neonato attraverso la scelta del nome sono, il più delle volte, dei rimandi sacri a Dio e alla grazia Divina.

L'approccio a un problema assai complesso come è la semantica dei nomi propri augurali comporta l'analisi di varie sfaccettature sia della semantica che dell'onomastica. Si tratta di un abbinamento di due discipline e dello studio del loro punto di convergenza. Di conseguenza, la prima parte del presente lavoro è una rassegna delle principali teorie e investigazioni riguardo all'esistenza o all'assenza di significato nei nomi propri. In un secondo momento metto in risalto le varie discipline - all'infuori della linguistica - per le quali il nome proprio rappresenta un punto di interesse e di studio. Si tratta del ruolo sociale dell'antroponimo, ma anche di campi di studio come la psicologia e la religione. Nell'ultima tappa della mia indagine mi soffermerò sul caso specifico dei nomi propri augurali, tanto dal punto di vista teorico quanto attraverso una rassegna dell' inventario onomastico augurale italiano contemporaneo.

Come accennato prima, il nocciolo duro dello studio dei nomi propri è rappresentato dai numerosi dibattiti relativi all'esistenza o all'assenza di significato negli 
antroponimi. Una cosa è certa: i nomi propri sono, all'origine, nomi comuni. Vivendo all'interno della comunità, c'è sempre stato l'imperativo che le persone si possano rivolgere l'una all'altra e possano parlare l'una dell'altra. Se la persona in questione era presente poteva essere indicata attraverso i gesti, la mimica ecc., ma in sua assenza era indispensabile che fosse differenziata dagli altri e questo si è realizzato attraverso il nome. Dunque, la necessità di comunicare ha portato all'apparizione dei nomi comuni, ma anche dei nomi propri, dato il fatto che i primi non sono riusciti a rispondere a tutte le esigenze dell'atto della comunicazione.

Pertanto, l'approccio ai nomi propri e, implicitamente, al loro significato, non si può verificare in modo isolato, ma deve essere collocato nel quadro assai più ampio della triade linguaggio - comunicazione - società. Di conseguenza, se si intende analizzare la semantica dei nomi propri, non ci si può limitare solamente ai campi dell'onomastica e della semantica, ma si deve ampliare l'area di ricerca fino a includere nell'analisi anche i campi della semiotica e della pragmatica. Pensiamo al punto di partenza della ricerca di Roman Jakobson (2002: 7): "il linguaggio è realmente il fondamento della cultura (...) il linguaggio infatti è lo strumento essenziale della comunicazione in quanto informazione". Parlando abbiamo sempre a che fare con due tipi di comunicazione: comunicare qualcosa a qualcuno e comunicarsi a qualcuno. Quest'ultimo processo rappresenta la comunicazione con un altro, una comunicazione che conta sull'altro, sulla sua presenza. Come risulta dall'opinione di Jakobson, il linguaggio è sempre per un altro, viene sempre usato per rivolgerci a un altro, anche quando questi non capisca ciò che è stato espresso. Ritroviamo facilmente in questa concezione il rispecchiamento del caso particolare degli antroponimi - la necessità dei nomi di identificare l'individuo per un altro anche se ciò che viene trasmesso attraverso il nome non è sempre trasparente e visibile al destinatario. Sulla stessa linea di pensiero, Eugen Coșeriu (2009: 51) sottolinea il fatto che il linguaggio non è semplicemente un fatto sociale, ma piuttosto "manifestazione primaria della società".

D'altra parte, l'aspetto creatore del linguaggio ha fatto sì che i segni venissero usati in un numero infinito di combinazioni al fine di esprimere significati. Usando le parole di Eugen Coșeriu (2009:41), "il parlare è linguaggio in quanto produzione concreta"; quindi lo studioso percepisce "il linguaggio in quanto attività creatrice". Nella sua capacità di parte integrante di qualsiasi lingua, il sistema onomastico usufruisce di questa caratteristica del linguaggio di essere produzione continua e infinita e, di conseguenza, vengono fuori costantemente antroponimi nuovi e diversi che arricchiscono l'inventario onomastico di tutte le lingue.

"Il carattere sociale dell'uso dei segni assicura il carattere più o meno costante del rapportare lo stesso segno allo stesso oggetto (classe di oggetti). Questa relativa costanza dell'uso sociale rende possibile il processo di significare - attraverso il linguaggio - degli oggetti, degli stati o degli eventi, e di conseguenza il comprendimento reciproco tra i parlanti della stessa lingua" (De Mauro 1978: 17), 
vale a dire che in questo modo viene assicurata l'interpretazione corretta del messaggio trasmesso, dunque una comunicazione efficace. Ma non tralasciamo un aspetto molto importante:

"una tradizione [del pensiero linguistico] plurisecolare ha quasi sempre perduto di vista che, in realtà, le forme linguistiche non hanno alcuna intrinseca capacità semantica: esse sono strumenti, espedienti, più o meno ingegnosi, senza vita e valore fuori delle mani dell'uomo, delle comunità storiche che ne facciano uso. In altri termini, l'errore sta nell'affermare e nel credere che le parole o le frasi significhino qualche cosa: solo gli uomini, invece, mediante le frasi e le parole, significano.

Non nelle forme linguistiche in se stesse, ma nelle società che le adoperano sta la garanzia del significare e del comunicare" (De Mauro 1965: 32).

\section{Similmente, Umberto Eco afferma che}

"un segno viene sempre costituito da uno (o più) elementi appartenenti ad un piano dell'espressione correlati in modo convenzionale con uno (o più) elementi appartenenti ad un livello del contenuto. Ogni volta che si verifica tale correlazione, riconosciuta da una società umana, si tratta di un segno. Solo in questo senso si può accettare la definizione di Saussure secondo il quale un segno è il corrispondente tra un significante e un significato (...) un'espressione non designa un oggetto, ma veicola un contenuto culturale" (Eco 1982: 65).

Ecco come si intrecciano gli elementi all'interno della menzionata triade. Di particolare interesse per il presente studio è il modo in cui le osservazioni generali sopraccennate attinenti al campo della semantica, della semiotica e persino della pragmatica si verificano anche per ciò che riguarda i nomi propri. Se si intende fare un'analisi della semantica dei nomi propri, non si può trascurare il contesto sociale e culturale. Nelle parole di Edoardo Sanguineti (1987: XI), "il nome è Cultura". L'uso degli antroponimi all'interno di una comunità con cui siamo familiari ci fa intravedere o almeno sospettare le ragioni originarie della scelta dei nomi in questione, ovvero il loro significato. Questa percezione è più sfumata o persino inesistente in una comunità di cui non conosciamo le realtà, le tradizioni, la cultura. I nomi di persona sono parte integrante della cultura che li ha generati e ne sono direttamente influenzati e foggiati.

Di seguito, farò una breve rassegna dei principali approcci riguardanti i nomi propri di persona lungo il tempo per poter, ulteriormente, presentare la mia ipotesi riguardante i nomi propri, con particolare riferimento agli antroponimi augurali. Intendo seguire le due direzioni definitorie per questo campo: quella dei descrittivisti oppure delle teorie del riferimento indiretto (come quelle di Frege, Russell, Searle) - secondo le quali i nomi propri designano un individuo attraverso un insieme di proprietà singolarizzanti che ne rappresentano il senso, e la direzione degli antidescrittivisti (Kripke, Donnellan ecc.) - secondo i quali l'antroponimo identifica in modo diretto e non mediato il suo referente senza ricorrere alle sue proprietà costitutive; si arriva, 
implicitamente, alla negazione dell'esistenza di qualsiasi valore semantico: i nomi considerati in quanto etichette prive di senso nell'opinione di J. S. Mill oppure il nome in quanto designatore rigido in conformità alle teorie di Kripke.

\section{L'approccio descrittivista}

I descrittivisti considerano che i nomi propri, così come anche le descrizioni definite, abbiano un senso. Più di tanto, il loro significato è più ampio, dato che consiste in certi rimandi ad una serie infinita di interpretanti che emergono in quanto molto più carichi di significato di quelli dei nomi comuni.

Gottlob Frege (1960: 23), nella sua teoria moderna del riferimento, definisce il nome in quanto significato e riferimento. Il significato di un nome, in questo caso, sarà la descrizione che i parlanti associano al relativo termine. Per essere più precisi, in base alla distinzione che fa tra significato e denotazione (riferimento), Frege considera il nome proprio da due prospettive: da una parte, il nome proprio designa un oggetto che rappresenta il suo riferimento o la sua denotazione, dall'altra, il nome esegue questo atto di riferimento in un modo determinato. Il punto di vista sotto il quale si presenta l'individuo costituisce il suo significato.

Bertrand Russel (1956: 31), d'altronde, ritiene che le descrizioni definite non hanno un riferimento, mentre il loro senso risiede nel verificarsi delle condizioni di verità delle relative descrizioni. Ciò nonostante, gli studi dei due ricercatori sono confluiti, la maggior parte delle volte, nella stessa direzione. Quindi, loro offrono una definizione comune del nome in quanto "descrizione definita e abbreviata o camuffata".

R. G. Heck (1995: 80) fornisce un altro approccio dei nomi propri. Lo studioso è dell'opinione che i nomi siano associati a certe descrizioni convenzionali che ad essi conferiscono il significato. Gli atti di comunicazione dall'esito favorevole che fanno uso di nomi propri richiedono non solo la trasmissione del referente del rispettivo nome, ma anche il senso adatto espresso attraverso quel nome. Di conseguenza, la conclusione di Heck è che si può trovare una spiegazione a certi fatti soltanto considerando la verità a seconda della quale ai nomi vengono associati significati specifici.

John Searle (1982: 215) propone la definizione del nome proprio in quanto disgiunzione logica di un numero determinato di descrizioni. Dato il fatto che un individuo è caratterizzato da una serie di tratti comuni identificabili anche in altri individui, ma la cui congiunzione non corrisponde se non all'individuo in questione, il senso del nome proprio è costituito da questa costellazione singolarizzante.

Il nome proprio si distingue dal nome comune perché fa riferimento ad un certo individuo (ed è questo il vero senso del nome proprio) e perché si foggia, per motivi religiosi e giuridici, sull'individuo che designa.

\section{L'approccio causale}

Per Saul Kripke la persona designata dal nome proprio rappresenta, nello stesso tempo, anche il suo riferimento. I vari aspetti presi in considerazione hanno condotto lo studioso verso l'identificazione di due termini: quello di designatore rigido, se in 
qualsiasi universo possibile esso designa lo stesso oggetto, e quello di designatore non rigido o accidentale, se non designa lo stesso oggetto in qualsiasi universo possibile. La fissazione del riferimento è opposta alla definizione di un termine in quanto avente il significato di un altro termine. Il nome, suggerisce Kripke, deriva da un "battesimo" iniziale all'interno del quale qualcuno dà un nome specifico ad un oggetto, sia indicandolo, sia facendo la connessione tra il nome e un set di qualità che considera possedute dal rispettivo oggetto. Da quel momento, il nome viene trasmesso da una persona all'altra, da un anello della catena all'altro. Nell'ambito di questo processo le proprietà che il parlante considera associate al nome finiscono per essere dissociate dall'oggetto. Il nome foggia l'oggetto in modo rigido, malgrado i suoi tratti caratteristici o le nuove scoperte ad esso relative. In altre parole, Mihaela Miron-Fulea (2005: 61), in linea con le osservazioni di Kripke, considera che

\begin{abstract}
"il nome proprio garantisce la continuità tra il mondo reale e gli universi possibili della logica modale: proprio quando costruiamo, a partire dal mondo reale, un mondo possibile, il referente rimane ancora l'individuo designato nel mondo reale, più di tanto, il nome proprio si manifesta anche attraverso il fatto che il suo riferimento non cambia, indifferentemente dalle variazioni dei predicati attribuiti al referente. La permanenza conferita al referente grazie al nome proprio va spiegata attraverso la catena causale che dirige l'attribuzione e l'uso del nome proprio. La sua origine viene collocata nell'atto sociale del battesimo attraverso il quale viene validato il suo funzionamento ulteriore".
\end{abstract}

In questo senso, Kripke (1982: 301) afferma: „Il nostro riferimento non dipende solo da quello che noi stessi pensiamo, ma dipende anche dalle altre persone della comunità, dalla storia della trasmissione del nome (proprio) fino a noi”.

Slavoj Žižek (1989: 21), analizzando la teoria di Kripke, sottolinea a sua volta il ruolo che viene attribuito alla comunicazione interpersonale. Nell'opinione del ricercatore, i descrittivisti mettono in risalto i contenuti interni e intenzionali della parola, mentre gli anti-descrittivisti considerano essenziale la catena causale esterna, il modo in cui una parola è stata trasmessa da un soggetto all'altro in virtù della tradizione. Žižek conclude che tanto i descrittivisti quanto gli anti-descrittivisti sono motivati dal desiderio di accertarsi dell'inviolabilità del punto cruciale, cioè la contingenza radicale del nome. In altre parole, loro intendono mostrare la necessità dei nomi per nascondere la verità secondo la quale il nome agisce in modo contingente e funzionale. Gli esempi che Žižek considera rilevanti sono le parole "democrazia" e "marxismo".

Come altri critici della sua teoria, anche Judith Butler (1993: 53) fa riferimento all'intenzione sociale omogenea che alla teoria di Kripke necessita. La suddetta teoria permette una trasmissione del nome che non crei problemi, che sia ben intenzionata e che renda impossibile l'uso scorretto del nome. Dal punto di vista di Kripke, deduce Judith Butler, sembra che qualsiasi utilizzo del designatore rigido presupponga l'esistenza di un parlante che sia stato iniziato in modo corretto riguardo l'uso del rispettivo nome e, di conseguenza, ne emerge una sorta di patto storico che in questo modo assicura una fissazione adeguata del nome. 
Josep Macìa (2004: 129), docente presso l'Università di Barcellona, fa un'analisi del ruolo comunicativo dei nomi propri. Lo studioso è interessato al ruolo che $\mathrm{i}$ concetti individuali complessi (o le idee) occupano all'interno degli atti di comprensione e utilizzo dei nomi propri. Macìa introduce due nuovi termini - coordinamento e necessità del coordinamento per combattere e per offrire delle soluzioni tanto nel caso delle teorie descrittiviste, quanto anche per ciò che riguarda quelle anti-descrittiviste. La teoria di Macì si costruisce intorno all'esempio di due villaggi i cui abitanti parlano la stessa lingua. Il concetto del loro essere coordinati per ciò che riguarda l'uso di un'espressione deve essere inteso nel senso che io sono coordinato ad un individuo A per ciò che riguarda il possesso da parte mia del nome $\mathrm{N}$ per riferirmi a $\mathrm{X}$ se e solo se io sono coordinato direttamente ad A oppure sono coordinato con qualcuno che è direttamente coordinato ad A.

Questa breve rassegna delle più importanti direzioni dello studio dei nomi propri intende provare il fatto che i dibattiti in questo senso non sono ancora conclusi e che, nelle parole di Edoardo Sanguineti (1987: VIII), „anche se posso scoprire l'origine di un nome proprio, quando è possibile, e sono in grado di arrivare ad un protosignificato, non gli potrò mai offrire una definizione accettabile".

Tranne i rimandi al senso e al riferimento dei nomi propri a cui si è già accennato, è mio desiderio illustrare nei prossimi paragrafi anche alcuni orientamenti dei ricercatori rumeni.

Victor Vascenco (1976: 66-101) fa un'analisi del senso referenziale, denotativo e connotativo dei nomi propri di persona. Lo studioso considera che il senso referenziale del nome ne rappresenti proprio il senso, essendo l'immagine concreta dell'oggetto a cui ci si riferisce e un'individualizzazione limitativa a carattere differenziato. Però il senso del nome non può essere stabilito senza ricorrere alla biografia delle persone dato che solo in questo modo si può fare una distinzione pertinente dal punto di vista delle realtà obiettive, dei nomi trasmessi da un referente all'altro. Per ciò che riguarda il senso denotativo, l'autore considera che, a patto che i nomi propri non siano nozioni o concetti, la loro denotazione è nulla. Invece, si può parlare di un senso connotativo degli antroponimi dato il fatto che esso fa riferimento al senso affettivo della parola, alle sue radici ancorate nell'immaginazione, nelle emozioni o nelle associazioni psicologiche. La conclusione del ricercatore è che gli antroponimi sono privi di senso denotativo, però questa carenza è compensata dagli altri due sensi che ne conferiscono anche il pregnante valore caratteriologico, presente soprattutto nei testi letterari.

Viorica Florea (1982: 78) afferma che si può parlare del senso del nome proprio solo se si considerano i due livelli: quello del parlato e quello della lingua. Di conseguenza, si può identificare un senso del nome proprio a livello del parlato (nell'ambito di un contesto specifico, quando il parlante conosce la rispettiva persona); questo senso viene proiettato anche a livello della lingua però solamente sotto la forma di un senso virtuale. A questo secondo livello il senso del nome traspare solo parzialmente sia dal genere del sostantivo (come, per esempio, Giovanni, Ion ecc. persona di genere maschile), sia quando si tratta di nomi propri a diffusione nazionale (come per esempio 
Eminescu ecc.) o internazionale (come per esempio Dante, Shakespeare ecc.). Nella visione della studiosa il senso del nome proprio non consiste in una nozione, ma in una somma di componenti semantici che designano le caratteristiche dell'oggetto tipo Shakespeare - "il più grande drammaturgo del mondo", fatto che dimostra la sua aderenza alle teorie descrittiviste.

In linea con le osservazioni di Viorica Florea secondo la quale a livello della lingua i nomi propri sono privi di contenuto, Augustin Pop (1987: 64) introduce la nozione di onomalinguaggio e cioè "un sottosistema della lingua che comprende tutto l'inventario delle parole che funzionano, ad un certo momento, in quanto nome proprio in una cultura". Nell'opinione dell'autore, solo a livello dell'onomalinguaggio i nomi propri possono avere un contenuto. Seguendo la distinzione che Frege fa tra senso e significato, Augustin Pop conclude che si può parlare del contenuto del nome proprio in quanto costituito da un significato relativamente stabile, identificatorio, referenziale, mentre il senso varia a seconda degli atteggiamenti del parlante nei confronti dell'oggetto che designa.

Un'osservazione di particolare interesse per il presente studio viene da parte di Ecaterina Mihăilă (1978: 267-278). La ricercatrice considera che l'apparizione di un nome proprio in una lingua coincida con la necessità dello sviluppo della sua funzione di identificazione. Così che, in un primo momento, il rispettivo nome esprime una qualità in modo generale grazie alla sua funzione perché ulteriormente, considerato in modo globale, diventi un marchio dell'oggetto. La perdita della motivazione, in seguito al consolidamento della funzione di identificazione lungo il tempo, è ancora più evidente per ciò che riguarda i nomi di persona, fatto dimostrato per esempio dalla scelta, al giorno d'oggi, del nome Andrei che esprime la qualità di "maschio, uomo", il cui significato viene trascurato o ignorato dalla maggior parte delle persone che lo usano. La scelta del nome si fonda, il più delle volte, su altre ragioni diverse da quella rappresentata dal suo significato. Dunque, considerati da un prospettiva sincronica, gli antroponimi si presentano completamente arbitrari rispetto al referente individuale. Il significato del nome proprio, nell'opinione di Ecaterinei Mihăilă, risiede nella sua motivazione ed è presente soltanto nel momento della sua genesi. D'altra parte, la studiosa richiama l'opposizione sistema (lingua) / uso (l'utilizzo del nome all'interno del sistema), aspetto che rimanda alla distinzione fatta anche da Viorica Florea tra i livelli della lingua e del parlato. Infatti, da questo punto di vista, le due ricercatrici arrivano ad una conclusione comune - nell'uso della lingua, il nome proprio si identifica con l'oggetto, in modo da esprimere l'infinità dei suoi tratti concreti. Ecaterina Mihăilă mette in risalto anche l'importanza di un delineamento chiaro dei vari livelli della lingua dato che soltanto essendone coscienti, siamo in grado di analizzare l'esistenza o l'assenza del senso del nome proprio. Conseguentemente, a livello della sua genesi il nome è motivato e ha senso; all'interno del livello funzionale in uso il nome si identifica con l'oggetto singolare conosciuto dal parlante, ricostruendo nella sua mente l'oggetto e le sue caratteristiche. 


\section{Tassonomia degli antroponimi}

Per essere in grado di analizzare i nomi propri augurali, essi devono essere prima collocati all'interno degli antroponimi in genere. Ecco la classificazione dei nomi propri di persona da varie prospettive:

a) etimologica - in questo gruppo sono inclusi:

- i nomi devozionali - nomi teofori, portatori di deità (it. Ezechiele 'in Dio la forza', Gabriele 'uomo di Dio' ecc.; ro. Isabela “promessa di Dio”, Lazăr "Elohim / Dio ha aiutato"), nomi di feste di carattere religioso (it. / ro. Natalia), nomi di santi oppure nomi calendaristici (it. Agata, Antonio ecc.; ro. Ion, Maria), nomi biblici (it./ro. Adamo, Eva / Adam, Eva);

- nomi affettivi - possono designare: l'ordine della nascita dei bambini all'interno di una famiglia (it. / ro. Settimo, Settimio / Septimiu, Ottaviano / Octavian), la gioia dei genitori per il nuovo nato, l'augurio per una vita piena di successo, il conforto morale ed economico che l'ultimo arrivato rappresenta per i genitori (i cosiddetti nomi augurali: it. / ro. Felicia, Gloria, Luciano / Lucian), tratti appartenenti alla fisionomia (i nomi descrittivi: it. / ro. Claudio / Claudiu “zoppo / șchiop"), elementi della natura e dell'universo (piante, animali, pietre preziose, fenomeni naturali ecc.: it. / ro. Iris / Iris, Viola / Violeta ecc.);

- nomi ammirativi - si tratta di antroponimi scelti a motivo dell'ammirazione per o a causa del prestigio di: una persona (un autore o un personaggio letterario, una personalità storica ecc.: it. / ro. Traiano / Traian), un popolo (gli esempi appartengono soprattutto alla categoria dei cognomi: it. Svevo, ro. Rus, Sârbu ecc.), una cultura (nomi ideologici: it. / ro. Madonna ecc.);

b) teorica e funzionale - di questa categoria fanno parte tanto le espressioni onomastiche che appartengono al sistema ufficiale di denominazione personale (nomi, cognomi), quanto quelle che appartengono al sistema popolare (nomignoli, soprannomi); viene incluso in questo gruppo anche il caso particolare degli pseudonimi;

c) semantica - questa prospettiva mira all'analisi della varie sfaccettature del nome proprio dal punto di vista del suo senso, come segnalato nella rassegna che precede. Se per ciò che riguarda i nomi e i cognomi ci sono stati numerosi dibattiti relativi al senso delle sopraccennate categorie, il significato è indubbiamente presente quando si tratta di nomignoli, soprannomi e persino pseudonimi. Mariana Istrate (2000: 93) fa una classifica degli pseudonimi dal punto di vista semantico, una tassonomia che, a nostro giudizio, può essere ampliata in modo da racchiudere tutti gli antroponimi. Di conseguenza, essi fanno riferimento in genera a:

- l'aspetto fisico se si considera la loro motivazione iniziale, dunque il loro significato etimologico. Si tratta di nomi come Paolo (it.) / Paul (ro.) - derivato dall'aggettivo paulus, piccolo;

- elementi caratteristici della personalità dell'individuo designato: Costante (it.) / Constanța (ro.) - dal latino constans, durevole, costante; Andrea (it.) / Andrei (ro.) - dalla 
parola greca che denominava il guerriero; Davide (it.) / David (ro.) - "essere amato"; Graziella (it.) / Grațiela (ro.) - "leggiadra, piacevole";

- mestieri o professioni: Camillo (it.) / Camil (ro.) - nome di origine orientale con il senso di "prete, partecipante a delle cerimonie speciali"; Fabian (it.) / Fabian (ro.) "colui che coltiva fave";

- l'origine dell'individuo che indica: Macedonio (it.) / Macedon (ro.) - "della Macedonia”; Cipriano (it.) / Ciprian (ro.) - "originario dell'isola di Cipro”;

- nomi di animali: Orso (it.) / Ursu (ro.) ecc.

Il nocciolo della mia ricerca si ritrova ovviamente a livello della categoria relativa all'etimologia, più esattamente nei nomi affettivi. Tranne i soprannomi, i nomi affettivi sono gli antroponimi che più conservano in modo visibile la loro motivazione originaria, e di conseguenza il loro senso.

Per inoltrarmi nell'approccio dei nomi augurali, prima si deve sommariamente delineare il ruolo dell'antroponimo in rapporto alla persona che esso indica. Anche se, il più delle volte, il nostro nome non è il risultato di una nostra scelta personale, ci rappresenta e ci identifichiamo nelle poche sillabe che lo compongono.

"Il nome personale è l'elemento primario dell'identità personale, ovvero il nostro Sé si
costruisce anche sulla base di questa unità nominale nella quale ci identifichiamo e ci
identificano, ci riconosciamo e siamo riconosciuti. Anche quando il nostro rapporto
con il nome è ambiguo oppure conflittuale, ad esso si attribuisce una funzione di con-
nessione tra noi e il mondo sociale: la nostra esistenza viene sintetizzata in un nome che
raccoglie l'inizio della nostra storia di vita, il suo evolversi, il suo stabilizzarsi e il suo con-
cludersi per diventare, successivamente, la nostra memoria storica" (Zara 2008: 320).

Il nome aderisce in modo assai stretto all'individuo da esso designato, lo connette al mondo esterno, gli rende possibile il contatto con altre persone. Il carattere sociale degli antroponimi non può essere trascurato dato che giocano un ruolo essenziale nell'esistenza collettiva. È questa una delle ragioni primordiali per cui la scelta del nome per il neonato non deve essere sottovalutata. Il nome sarà uno dei numerosi ma essenziali elementi che compongono, costruiscono un'identità. Sarà un punto di contatto con gli altri e, di conseguenza, influirà in una certa misura sul destino della persona da esso indicata. Frequentemente vengono segnalati casi di persone che hanno desiderato cambiare il loro nome a causa del disagio che ne sentivano usandolo e dell'impatto sociale sgradevole che esso generava.

"Il nome personale appare svolgere un compito esistenziale significativo: esso contiene la storia di vita della persona, trasporta la biografia vivente della persona, sintetizzandola in un'identità nominale, diventa una memoria familiare, personale e sociale. Conseguentemente il nome diviene uno dei primi elementi di riconoscimento del Sé all'interno del mondo sociale e (...) appartiene al complesso dei beni culturali, sociali e spirituali di un popolo" (Zara 2008: 329). 
La ricercatrice Georgia Zara ha approcciato il nome proprio dal punto di vista psicologico. Riportiamo, di seguito, l'opinione della studiosa rumena Tatiana Petrache che ce lo presenta da un'altra angolatura, quella religiosa:

"Invocare il nome di una persona significa farla presente in modo effettivo. Fare una cosa a nome di qualcuno o chiamare qualcuno con il suo nome sono degli atti che presuppongono un rapporto dialogico, diretto. Il nome è la chiave della persona; menzionare il nome di qualcuno significa collocarti nella sua presenza" (Petrache 2016: 7).

Se per i primitivi il nome era magico, aveva il potere di proteggerli da infortuni o incantesimi, il nome all' interno del cristianesimo non esercita più il fatale determinismo nei confronti del destino umano.

"Il nome di un santo oppure un nome che invoca Dio è privo di significato e forza in assenza di una partecipazione cosciente di colui che investe la realtà invocata. Invocare il nome di Dio comporta essere propensi al Suo amore, sacrificarsi per Lui" (Petrache 2016: 8).

Se non si è consapevoli del ruolo che il nome occupa a livello del nostro rapporto con la divinità, l'antroponimo diventerà una semplice etichetta che identifica la persona. In tal caso, il nome non comporta più l'obbligo della responsabilità nei confronti di Dio e degli altri. Attraverso il battesimo l'essere umano entra in un rapporto dialogico con la divinità,

"ma solo quando il nome è principio distintivo e formatore, solo allora esso rappresenta una profonda forza di personalizzazione. La persona riceve un nome proprio e sa che, allorquando viene chiamata con questo nome, si mira il suo intero essere e deve rispondere con il suo intero essere" (Stăniloae 1978: 53).

In sostanza, l'antroponimo nella concezione cristiana è uno strumento che foggia la personalità della persona designata ed è un ricordo costante del rapporto speciale, personale, unico di ognuno di noi con la divinità.

Ecco il modo in cui due visioni appartenenti a campi di studio diversi, convergono verso un'unica direzione: l'importanza del nome, puntando sul ruolo centrale, essenziale che esso occupa nell'esistenza di ognuno di noi. Lucian Blaga (1969: 276) accomuna le due posizioni indicate sopra in una sublime definizione del nome in quanto "metafora rivelatrice".

Mettiamo in risalto ancora una volta il peso della scelta del nome. "I nomi sono rappresentativi dello status socio-culturale, della moda, dei gusti, delle emozioni e aspettative che si ripongono sul bambino al quale si decide di assegnare un certo nome oppure un altro" (Zara 2008: 334). La motivazione che conduce alla scelta di un nome può essere di carattere religioso, può integrarsi in una tradizione familiare $o$ 
locale, nel folclore di una particolare regione, oppure può essere dettata dal desiderio di unicità o dall'estetismo. La scelta del nome, benché orientata sul figlio, riflette sempre la personalità e la cultura dei genitori anche se impegnati a rispettare i futuri interessi del figlio. Come già accennato, gli antroponimi sono stati creati dai nomi comuni, godendo di un valore significativo per il destino e i tratti caratteristici della persona da essi designata: si tratta di nomi con riferimento diretto alla divinità o alle forze soprannaturali, nomi dedicatori - che indicano che il figlio è dedicato a Dio, nomi teofori contenenti il nome di Dio, nomi augurali o gratulatori, che esprimono un augurio per il denominato o un ringraziamento a Dio per il figlio che ha concesso. Questi ultimi venivano scelti in virtù della loro capacità ipotetica di foggiare l'esistenza del bambino in conformità al significato del rispettivo nome. Ecco il fenomeno dell'emergenza dei nomi propri augurali medievali come viene esposto dallo studioso Enzo Caffarelli (2013: 52):

"Dopo il 1000, e specie dal XII secolo, lo stock onomastico - ossia il repertorio a disposizione delle famiglie per dare un nome ai figli - si era drasticamente ridotto. Della classicità latina ci si era quasi dimenticati. I santi venerati erano un numero assai ristretto (...) Anche i nomi dei sovrani popolari in tutta l'Europa si potevano contare sulle dita di una mano (...) In attesa dei grandi santi degli ordini mendicanti (...) il popolo s'inventò nomi trasparenti, gratulatori e augurali”.

Lo studioso fa l'esempio di nomi come Amadeo, Graziadei, Salimbene; si tratta anche di nomi soprattutto descrittivi come Allegro, Fiore, Grazia; altri illustravano la gioia di completare la famiglia con il nuovo arrivato: Bencivenga, Benvenuto, Bonaiuto. Di particolare interesse è il caso di un gruppo specifico di nomi che rammentavano alla famiglia sia di aver perso un figlio e di essere grati di averne ricevuto un altro, sia di una grave malattia e del recupero augurato all'infermo. Si tratta di nomi come Tornabene, Ristoro, Rimedio, Ritrovato. "Così la famiglia esprimeva, attraverso l'onomastica, la sua soddisfazione, anche misurando il valore della sostituzione: Paribono, Paribello, Buoncambio, addirittura Migliore. Da cui i cognomi Cambi, Parboni, Migliorini" (Caffarelli 2013: 53). E infatti, anche se per ciò che riguarda la maggior parte dei nomi augurali al giorno d'oggi non si può più parlare di trasparenza del significato, di visibilità della motivazione originaria, come nemmeno di alta frequenza, restano comunque di particolare interesse per i ricercatori, data la loro conservazione in quanto cognomi. Di solito non si incontrano in quanto nomi, ma hanno arricchito il repertorio dei cognomi e restano in uso all'interno della lingua. Però, velate sotto questa forma, quante persone fanno ancora attenzione al significato e alla provenienza di tali nomi? Quanto è ancora trasparente e chi avverte ancora la loro motivazione primaria? Chi pensa, sentendo cognomi come Malavolta, Soverchia, Orcibasta ecc., che ad un certo punto nel passato c'è stato un antenato che la famiglia aveva voluto che fosse l'ultimo nato? "Uno solo tra questi <<nomi del disappunto >> è sopravvissuto fino al Novecento, quasi soltanto in Toscana: Finimola, ossia smettiamola qui, tu sarai l'ultima. Se incontrate il figlio 
o la figlia di una signora Finimola, chiedetegli/le quanti zii abbia" ci consiglia Enzo Caffarelli (2013: 54).

Analizzando le principali teorie riguardanti il senso dei nomi propri per poi proiettarle sul quadro specifico dei nomi propri augurali, viene validata, nella nostra opinione, l'ipotesi che i nomi augurali trasmettono un messaggio rivelatore riguardo alla persona designata. Gli antroponimi sono in grado di rivelare (a chi fa attenzione) il ceto sociale, la provenienza, $\mathrm{i}$ fattori socio-culturali che hanno influito sullo sviluppo di una persona. Più di tanto, i nomi augurali sono allusivi al desiderio, al timore oppure alle speranze dei genitori per il neonato. Il più delle volte, la loro scelta è stata motivata da un pericolo, una malattia o simili. A nostro parere, il senso dei nomi propri augurali risiede nel messaggio che si è voluto trasmettere attraverso la loro scelta, l'augurio, il desiderio di godersi la vita, la famiglia, la salute, la bellezza ecc. e di richiamare, a tal fine, l'attenzione e l'aiuto della Divinità. A differenza dei nomi laici, la maggior parte degli antroponimi augurali presuppone o comunque coinvolge la grazia divina nella cui assenza non ci sarebbe neppure il motivo di tale gioia - cioè la nascita del nuovo membro della famiglia.

Anche se il repertorio dei nomi augurali nell'uso odierno non è più così ricco come era nell'antichità e nell'epoca medievale, la loro penetrazione nel sistema ufficiale, in quanto cognomi, ci offre ancora la possibilità di analizzarli ma anche, non meno importante, di goderceli. La migrazione della categoria dei nomi alla categoria dei cognomi è avvenuta lungo vari decenni, in alcuni casi persino secoli. Una volta persa la trasparenza del nome augurale, la sua scelta non si giustificava più. Però, questo fatto non ha portato alla sparizione definitiva dei rispettivi nomi, grazie al fatto che tanti sono diventati cognomi. Di conseguenza, si sono conservati lungo il tempo e sono identificabili e presenti nel repertorio onomastico contemporaneo.

Eppure, la scelta dei nomi augurali nell'epoca contemporanea non è sparita del tutto. Si possono ancora identificare nomi augurali attualmente in uso, alcuni essendo persino di media o alta frequenza. Ne segnaliamo alcuni ${ }^{1}$ :

- Abbondanza (s.f., frequenza: 1212) che continua i nomi augurali latini di epoca cristiana Abundans, Abundantia, formato dal part. pres di abundare "abbondare";

- Alba (s.f., frequenza: 30789) dal nome di tipo augurale Alba;

- Allegra (s.f., frequenza: 521) dal latino - alacer "allegro, gioioso, vivace";

- Amore (s.m., frequenza: 143);

Amatore (s.m., frequenza: 303);

Amato (s.m., frequenza: 2985);

Amata (s.f., frequenza: 384 );

- Bellino (s.m., frequenza: 444) dal soprannome o nome Bello / Bella, come costatazione o augurio di bellezza per il figlio;

1 La fonte di documentazione per gli esempi elencati è il dizionario I nomi di persona in Italia: dizionario storico ed etimologico di Alda Rossebastiano e Elena Papa pubblicato nel 2005. Il periodo preso in considerazione per valutare la tradizione e la frequenza dei nomi individuali menzionati è compreso tra il I gennaio 1900 e il 31 dicembre 1994. 
Bellina (s.f., frequenza: 232);

- Benigno (s.m., frequenza: 1390) nome augurale latino con il significato di "benigno, benevolo, buono"; sostenuto ulteriormente dall'agionimo, San Benigno;

Benigna (s.f., frequenza: 545);

- Bonaventura (s.m., frequenza: 2282) dal nome augurale e gratulatorio Bonaventura "che abbia, che costituisce (per la famiglia) una buona fortuna; sostenuto ulteriormente dall'agionimo;

- Bonifacio (s.m., frequenza: 1090) nome augurale formato da "bonum" e "fatum", con il significato quindi " (che abbia) una buona sorte"; sostenuto ulteriormente dall'agionimo;

- Desiderato (s.m., frequenza: 108) per esprimere il desiderio e l'augurio di salvezza spirituale, o la gratitudine per la nascita di un figlio desiderato;

Desiderio (s.m., frequenza: 1958);

Desiderata (s.f., frequenza: 285);

- Fiore (s.m., frequenza: 4544) da "fiore" come nome augurale;

Fiorella (s.f., frequenza: 35710 );

Fiorenza (s.f., frequenza: 14109);

Fiorenzo (s.m., frequenza: 15448);

- Florina (s.f., frequenza: 380) dal latino Florus e dal suo derivato Florius, connessi con la dea della primavera, dei fiori e della vegetazione [e quindi augurale];

- Fortunato (s.m., frequenza: 21782) nel senso augurale di "buona fortuna, destino favorevole";

Fortunata (s.f., frequenza: 17368);

- Gemma (s.f., frequenza: 34118) dal latino gemma "pietra preziosa", dato a una figlia considerata "cosa bella, cara o preziosa";

- Gioia (s.f., frequenza: 4388) dal nome augurale gioia;

- Perla (s.f., frequenza: 1366) come nome augurale: "bella, cara come una perla";

- Prospero (s.m., frequenza: 3235) come nome augurale "prospero, felice, fortunato";

Prospera (s.f., frequenza: 616);

- Regina (s.f., frequenza: 22532);

- Stella (s.f., frequenza: 28549) dato a una bambina che è o ci si augura che sia bella come una stella;

- Ventura (s.f., frequenza: 482) in quanto augurio per una sorte favorevole;

- Vitale (s.m., frequenza: 3119 ) che dà, che ha vita; pieno di vita, che vivrà bene;

- Vittoria (s.f., frequenza: 84799) dall'augurale latino derivato dal verbo vincere;

- Viviano (s.m., frequenza: 717) dal nome Viviano, che continua il cognome latino Vivianus, certamente connesso alla radice viv-di vivere;

Viviana (s.f., frequenza: 26045).

La conclusione che si può trarre da questi risultati è il fatto che al giorno d'oggi gli antroponimi augurali non sono così diffusi come nel passato. La ragione per cui 
sono caduti in disgrazia dipende probabilmente dal fatto che sono diventati opachi nel corso del tempo e hanno perso la loro trasparenza. Conseguentemente, si è persa la motivazione che giustifica la loro scelta. Un altro motivo della loro scarsa diffusione è dovuto all'influenza della moda. Ai nostri giorni i genitori puntano più sull'estetismo che sul senso degli antroponimi. Infatti, a mio giudizio, l'epoca moderna favorisce prevalentemente i nomi-etichetta invece di quelli carichi di significato. Se invece l'antroponimo viene scelto proprio grazie al suo significato originario (come succede nel caso dei nomi augurali), allora è ovvio che, attraverso quel nome, si trasmette un messaggio, si allude ad aspetti ancora da svelare e da scoprire.

Cristallizzatisi lungo i secoli, gli antroponimi hanno perso il loro valore primitivo di evocare una persona in virtù della loro motivazione originaria. Del senso iniziale ci resta solo un riflesso, un eco dell' intenzione suggestiva primordiale. In alcuni casi anche nell'epoca moderna la trasparenza del nome, vale a dire un rapporto con un termine significativo della lingua, può essere un motivo della scelta, come nel caso dei nomi augurali sopra elencati. In tal caso, a nostro giudizio, la scelta è di importanza basilare e rappresenta una chiave di interpretazione della persona indicata. Ci sono anche dei nomi che sono diventati semplici etichette per aver perso il loro valore semantico. Tatiana Petrache (2016: 14) fa l'esempio del nome di origine latina Camil, Camila, (it.) Cam(m)illo, Cam(m)illa, Camelia, (ro.) Camil, Camelia. Alle origini, si trattava degli adolescenti che assistevano i sacerdoti durante i sacrifici pagani, però oggi si è perso il significato e il nome è sopravvissuto soltanto grazie alla moda. Dal punto di vista semantico, l'antroponimo è ormai privo del suo significato primario e rappresenta un semplice marchio per poter distinguere gli individui all'interno di un gruppo. Il nome non è più compreso oggigiorno dal punto di vista etimologico. Tale trasformazione $\mathrm{e}$ altre simili si collocano nell'ampio quadro dell'onomastica europea odierna e riflettono l'evoluzione interna e sociale dell'essere umano moderno.

\section{Bibliografia}

Alinei, Mario, Francesco Benozzo. 2017. Dizionario etimologico-semantico dei cognomi italiani DESCI. Savona: PM Edizioni.

Blaga, Lucian. 1969. Trilogia culturii. Geneza metaforei și sensul culturii III: 275-290. București: Editura pentru Literatură Universală.

Butler, Judith. 1993. Bodies that Matter: On the Discursive Limits of „Sex”. New York: Routledge. Caffarelli, Enzo. 2013. Dimmi come ti chiami e ti dirò perché: storie di nomi e di cognomi. Bari: Editori Laterza.

Coseriu, Eugenio. 1997. Linguistica del testo. Roma: La Nuova Italia Scientifica.

Coșeriu, Eugen. 2009. Omul și limbajul său. Studii de filozofie a limbajului, teorie a limbajului și lingvistică generală. Iași: Editura Universității “Al. Ioan Cuza”.

De Felice, Emidio. 1982. I nomi degli italiani. Informazioni onomastiche e linguistiche, socioculturali e religiose. Rilevamenti quantitativi dei nomi personali dagli elenchi telefonici. Venezia: Marsilio Editori.

De Felice, Emidio. 1986. Dizionario dei nomi italiani. Milano: Mondadori. 
De Felice, Emidio. 1987. Nomi e cultura: riflessi della cultura italiana dell'Ottocento e del Novecento nei nomi personali. Venezia: Marsilio Editori.

De Mauro, Tullio. 1965. Introduzione alla semantica. Roma-Bari: Editori Laterza.

De Mauro, Tullio. 1978. Introducere în semnatică. București: Editura Științifică și enciclopedică. Eco, Umberto. 1982. Tratat de semiotică generală. București: Editura Științifică și enciclopedică. Florea, Viorica. 1982. Nivelul vorbirii şi al limbii la numele proprii. Studii de onomastică 3: 74-86. Frege, Gottlob. 1960. On Sense and Reference. Oxford: Blackwell.

Heck, R.G. Jnr. 1995. The Sense of Communication. Mind 104 (413): 104-112.

Istrate, Mariana. 2002. Numele propriu in textul narativ. Cluj-Napoca: Napoca Star.

Jakobson, Roman. 2002. Saggi di linguistica generale. Milano: Feltrinelli Editore.

Kripke, Saul. 1982. La logique des noms propres. Paris: Minuit.

Kripke, Saul. 2001. Numire şi necesitate. Bucureşti: Editura All Educational.

Lévi-Strauss, Claude. 1962. La pensée sauvage. Paris: Plon.

Macìa, Josep. 2004. Proper Names: Ideas and Chains. Canadian Journal of Philosophy 30: 129-155.

Mihăilă, Ecaterina. 1978. Despre geneza şi funcția numelor proprii. Limba română XXVII (3): 267-278.

Miron-Fulea, Mihaela. 2005. Numele proprii. Interfața semantică-sintaxă. Bucureşti: Editura Universității din Bucureşti.

Oltean, Ștefan. 2006. Introducere în semantica referențială. Cluj-Napoca: Presa Universitară Clujeană.

Petrache, Tatiana. 2016. Dicționar de nume. Iași: Editura Doxologia.

Pop, Augustin. 1987. Numele propriu - un paradox semantic. Studii de onomastică IV: 61-67.

Rossebastiano, A., E Papa. 2005. I nomi di persona in Italia: dizionario storico ed etimologico. Torino: UTET.

Russell, Bertrand. 1956. On Denoting. London: George Allen and Unwin.

Sanguineti, Edoardo. 1987. La missione del critico. Milano: Marietti.

Searle, John. 1982. Sens et expression. Paris: Minuit.

Searle, John. 1983. Intentionality. An Essay in the Philosophy of Mind. Cambridge: Cambridge University Press.

Stăniloae, Dumitru. 1978. Teologia Dogmatică Ortodoxă 3. Bucureşti: Editura Institutului Biblic și de Misiune al Bisericii Ortodoxe Române.

Ullmann, S. 1952. Précis de sémantique française. Berna: A. Francke.

Vascenco, Victor. 1973. Asupra sistemului standard al numelor de familie româneşti (reflectat în antroponimia bucureşteană)". Cercetări lingvistice 24 (3): 265-277.

Vascenco, Victor. 1976. Sens referențial, sens denotativ, sens conotativ. Cu privire specială asupra onomasticii. Studii de onomastică I: 66-81.

Zara, Georgia. 2008. La psicologia del nome personale. La vita quotidiana come laboratorio di psicologia sociale: 320-341. Milano: Giuffrè Editore.

Žižek, Slavoj. 1989. The Sublime Object of Ideology. London: Verso. 